\title{
Cipango
}

Cahiers d'études japonaises

$24 \mid 2021$

Eugénisme dans le Japon moderne et contemporain

\section{Sous la plume des mineurs : Sākuru-mura}

Under miners' pen: Sâkuru-mura

\section{Makiko Andro-Ueda}

\section{OpenEdition}

Journals

Édition électronique

URL : https://journals.openedition.org/cipango/4693

DOI : $10.4000 /$ cipango.4693

ISSN : 2260-7706

Éditeur

INALCO

Édition imprimée

Date de publication : 20 octobre 2021

Pagination : 211-235

ISBN : 9782858313969

ISSN : $1164-5857$

Référence électronique

Makiko Andro-Ueda, «Sous la plume des mineurs : Sākuru-mura », Cipango [En ligne], 24 | 2021, mis en ligne le 11 octobre 2021, consulté le 09 avril 2022. URL : http://journals.openedition.org/cipango/4693 ; DOI : https://doi.org/10.4000/cipango.4693

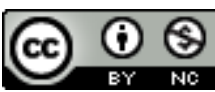

Cipango est mis à disposition selon les termes de la Licence Creative Commons Attribution - Pas d'Utilisation Commerciale 4.0 International. 


\title{
Sous la plume des mineurs : Säkuru-mura
}

\author{
Under miners' pen: Sâkuru-mura
}

\author{
Makiko Andro-Ueda \\ Mon bras creusé par un morceau de charbon est guéri. \\ Sur la cicatrice de la chair déborde \\ Telle une matière étrangère ${ }^{1}$
}

\section{Sākuru-undō et Sākuru-mura}

Les années 1950 furent une décennie au cours de laquelle le peuple japonais s'efforça de s'approprier les nouveaux droits civiques acquis à la suite de la défaite et de conférer un sens au fait de désormais considérer tout individu comme sujet. Ce tâtonnement, motivé par une aspiration à de nouveaux rapports sociaux et par une ouverture à des pratiques culturelles jadis interdites ou inaccessibles, a pris la forme de diverses activités associatives, dites Säkuru-undō (サークル運 動 mouvements de cercle). Säkuru, que nous traduisons provisoirement par cercle, désigne tout groupe ou association de personnes dont les membres adhèrent par choix. Cette liberté du mode d'adhésion distingue les cercles des organisations

1. 炭塊にえぐられて癒えし吾の腕に異質の如く盛り上がる肉あり Tankai ni egurarete ieshi ano udeni ishitsuno gotoku moriagaru niku ari : tanka publié par le mineur Yamamoto Tsuguru 山本詞 dans une des livraisons du Village des cercles. 


\section{CIPANGO}

212 Cahiers d'études japonaises nº 24

professionnelles ou des groupes fondés sur un lien de parenté ou de proximité de résidence ${ }^{2}$.

La transition du charbon vers le pétrole a engendré d'importants mouvements sociaux dans les mines de Mitsui-Miike en 1953 et en 1959, suivies ensuite par plusieurs mines moins importantes du nord de Kyūshū, mais l'expressivité des mineurs ne se limitait pas au domaine des luttes sociales. Dès le lendemain de la défaite, comme partout ailleurs, des centaines de cercles ont vu le jour dans les bassins houillers ${ }^{3}$. Grâce à l'un de ces mouvements, des facettes de la vie de Chikuhō en déclin nous sont demeurées accessibles. Il s'agit de nombreux textes, de la main principalement de travailleurs de secteurs divers, donnés à la revue Säkuru-mura (サークル村Le Village des cercles), sous forme de poésies, journaux, débats ou encore de différents récits ${ }^{4}$. La revue fut publiée entre septembre 1958 et octobre 1961, à Nakama, un hameau de mineurs situé dans le nord du département

2. Bien que plusieurs auteurs suggèrent une analogie avec les kō (講), associations religieuses ou profanes qui se sont développées depuis le moyen âge, par exemple Tsurumi Shunsuke 鶴見俊輔 (Tsurumi, 1976, p. 5-7), les modalités du cercle semblent avoir été perçues comme celles d'un mouvement moderne. La première occurrence du terme dans le sens d'association remonte à 1931, sous la plume de Kurahara Korehito 蔵原惟人 (1902-1991), l'un des principaux théoriciens de la Fédération nationale prolétarienne pour l'art (全日本無産者芸術連盟 Zen-nihon musansha geijutsu renmei). De retour de Moscou, ce militant proposa de créer « des cellules à vocation culturelle dans les entreprises industrielles, selon un modèle d'inspiration soviétique » (YUCHI, p. 405). Le mot désignait des associations culturelles, surtout littéraires, organisées par des employés d'entreprise. Le cercle changera de nature après la Défaite. La sociologue Amano Masako décrit l'ampleur du phénomène des cercles entre 1945 et 1955 en ces termes : « Lors de la Défaite (...), (1)'une des forces ayant contribué à définir l'air du temps était la démocratie à l'américaine, l'autre était le marxisme qui a eu un regain d'influence. Le poids de ce dernier était particulièrement important, entraînant un climat de discorde entre d'un côté les cercles et, de l'autre, les partis politiques et les syndicats qui cherchaient à exercer sur eux une influence » (AMANO, p. 21).

3. Mizutamari (2013, p. 39-42) situe le temps fort des cercles dans le secteur du charbon entre 1954 (après le tassement des effets de la Purge rouge) et la fin des années 1950 (avec le déplacement de la vocation des cercles du domaine social vers celui de la consommation).

4. Seuls manquaient comme genres courants les haikus, certainement trop courts pour rendre compte d'une situation sociale, et les romans, trop longs à produire et difficiles à diffuser dans ce cadre. 
de Fukuoka. En trois ans, trente-quatre numéros furent publiés, avec un tirage d'environ mille exemplaires. Le choix de Chikuhō était stratégique pour les jeunes fondateurs de la revue, car il reflétait l'importance du symbolisme du charbon à cette époque 5 . Ils ont choisi de s'implanter à Nakama, où était déjà installé l'un d'eux, Ueno Eishin 上野英信 (19231987). Après la défaite et son irradiation à la bombe $\mathrm{H}$, celui-ci avait abandonné sa vie d'étudiant brillant à l'université de Kyōto pour « prendre la revanche de Hiroshima en devenant mineur ${ }^{6}$ ».

5. Un jeune mineur décrit de la manière suivante l'atmosphère qui régnait dans le groupe et la place particulière réservée aux travailleurs du charbon : «on m’a donné les surnoms de "parleur grandiose", "Don Quichotte", "gaillard", qui semblent être aussi des dénominations des travailleurs du charbon. Je les accepte avec gratitude, mais les membres du Village, fiers de leur goût pour la causticité, pourquoi me parlent-ils avec une politesse qui déroute un peu, sentimentalement, comme s'ils me regardaient de travers ? avec un artifice de... paysans ?「大ぶろしき」で「ドン・キホーテ」で「陽気な野郎」 で、とはサークルの皆さんが私にくださったタイトルだ。と同時に炭鉱労 働者にいってる言葉とも受け取れる。どれについても私は有難く頂戴して いるわけだが、それにつけても毒舌を持って任じるサークルの皆さんが、 あんがいインギンに、しんみりと、横目でものを言うのが気にくわないの だ。言うなれば、農民的ずるさ、とでもいうか。 (S-m, 2-4-25)». Travailler dans le secteur houiller en 1960 devait susciter auprès des autres une gêne, comme le ferait malgré lui quelqu'un atteint d'une maladie incurable. La revue organisera la visite des galeries pour des membres non mineurs. (《Bokutachi wa yami ni attekita »ぼくた ちは闇に会ってきた [Nous avons été rencontrer les ténèbres], S-m, 1-3-35/41).

Désormais, lorsque nous citons la revue Sākuru-mura, nous indiquons la référence dans le corps du texte entre parenthèses, avec le sigle « $S$ - $m$ » suivi du numéro indiquant l'année de publication, du numéro du volume et de la page, en les attachant par un tiret. Le premier chiffre (le numéro correspondant à l'année) se renouvelle à chaque début d'année. Ainsi, le chiffre 1 indique qu'il s'agit d'un numéro de l'année de lancement, à savoir 1958. Lorsque la citation s'étale sur plusieurs pages, nous indiquons les numéros des première et dernière pages séparées par une barre oblique. Par exemple, (S-m, 3-3-23/27) renvoie aux pages 23-27 du numéro 3 de l'année 1960. Le succès « posthume » de la revue illustre l'importance du symbolisme du charbon tout au long des années d'après-guerre.

6. Ueno se préparait à intégrer l'élite de l'administration japonaise en Corée, persuadé de l'idéal de l'Union de cinq races (五族協和 gozoku-kyōwa). Après la défaite, la conscience d'avoir été trahi, la culpabilité et la haine de l'Amérique, l'ont empêché de se réapproprier la vie qui lui avait été destinée. C'est en travaillant sous terre et en découvrant la solidarité des ouvriers contre les accidents, écrit-il, qu'il redécouvre l'humanité : « Tout ce que je voulais c'était effacer Hiroshima de mon esprit. Je ne pouvais vraiment pas continuer à 
CIPANGO

214 Cahiers d'études japonaises nº 24

\subsection{Le contexte}

Cette édition plutôt éphémère, issue d'un endroit si reculé, a toutefois « exercé une forte influence dans les années $1960 \gg$ (Matsubara, p. 5), notamment sur la pensée de certains intellectuels, tel Tsurumi Shunsuke 鶴見俊輔, ou sur celle des acteurs de la nouvelle gauche. On peut légitimement la qualifier d'expérience d'envergure pour une émancipation humaine, partant de motivations similaires à celles qui avaient cours à la capitale avec le « débat sur la subjectivité (主体性論 争shutaisei-ronsō) 》. Depuis le début des années 2000, le phénomène des cercles dans sa diversité, et en particulier Säkuru-mura, connaît un important regain d'intérêt chez les chercheurs ${ }^{7}$. On s'y intéresse en tant que mouvement social, ou encore pour les textes signés par des personnages centraux qui ont marqué la mentalité des années 1960. En revanche, peu de chercheurs ont porté leur attention sur la part exacte des mineurs ou des anciens mineurs, ni sur la nature de leurs contributions. Esquissons ici une présentation de Säkuru-mura sous cet angle, en éclairant les événements et les écrits directement liés aux métiers du charbon (soit thématiquement, soit par l'identité de l'auteur). Les mineurs, en effet, n'étaient pas symboliquement, ou passivement « mobilisés » par le simple appel des organisateurs. La revue était éditée par le « Groupe de recherches sur les cercles de Kyūshū (九州サークル研究会 Kyūshū sākuru kenkyū-kai) », fondé par trois personnes : Tanikawa Gan 谷川雁 (1923-1995), Morisaki Kazue 森崎 和江 (1927-) et Ueno Eishin. Ils avaient déjà une vocation bien établie d'écriture et publieront leurs œuvres significatives durant cette période. Tanikawa Gan, poète et pivot du mouvement, sortait d'une longue période où il avait été soigné

vivre sans effacer le spectacle de l'enfer des vivants, si atroce qu'un humain ne doit pas le voir. Que serais-je devenu si les ténèbres de Chikuhō n’avaient pu m’envelopper alors ?» 私はただやみくもに、心からヒロシマを消したかっただけである。あの、 人間が見てはならない凄絶な生地獄の光景を消さなければ、到底、生きて 行かれなかったのである。もし、あの時筑豊の闇が私をつつんでくれなか ったら、私は果たしてどうなっていたことか。(ARAKI, p. 15).

7. Pour ce qui est de Sākuru-mura, l'ouvrage de Matsubara Shin.ichi intitulé Gen.ei no komyūn : Säkuru-mura kenshō-suru 幻影のコミューン サークル村を検証する [La commune en mirage : examiner Le Village des cercles] posera en 2001 le fondement proprement dit des recherches. La revue sera intégralement rééditée en 2006. 
pour la tuberculose, qui fit suite à son licenciement du journal Nishi-nihon shinbun 西日本新聞 pour ses activités de militant communiste. Morisaki Kazue, née dans la Corée occupée et rapatriée, était une jeune mère qui composait des poèmes. Ueno Eishin, ancien soldat et irradié de Hiroshima, était devenu mineur à Nakama. Plus de deux cents trente personnes ont collaboré à la revue, majoritairement des militants actifs de divers cercles et de syndicats d'entreprise, ces derniers appartenant à des fédérations nationales : le secteur du charbon bien entendu, mais également ceux de la sidérurgie, de la poste, des collectivités territoriales ou de l'éducation nationale, etc. On ignore la proportion exacte des mineurs, car les contributeurs n'ont pas précisé leur métier, sauf pour les textes de teneur militante ou de débats.

\subsection{La publication}

Le but du «Groupe de recherches sur les cercles de Kyūshū », se définissant lui-même comme cercle, était de « créer un village » en mettant en contact l'ensemble des cercles du Kyūshū et de la région orientale de l'île de Honshū (département de Yamaguchi) pour les dynamiser en suscitant des débats entre eux (S-m, 1-1-4). La direction considérait les conflits internes (内部抗争 naibukōsō) comme une nécessité pour un bon fonctionnement du groupe : « les violentes failles et ruptures entre ouvriers et agriculteurs, intellectuels et peuple, générations ancienne et nouvelle, centre et périphérie, hommes et femmes, d'un domaine à l'autre, ne sauraient être dépassées que par l'unification obtenue grâce aux oppositions, affrontements houleux, éclaboussant tout autour, ainsi qu'une osmose (交流 kōryū) de grande envergure » $(\mathrm{S}-\mathrm{m}, 1-1-3)^{8}$. Il est également à

8. 労働者と農民の、知識人と民衆の、古い世代と新しい世代の、中央と地 方の、一つの分野と他の分野の間に横たわる激しい断層、亀裂は波瀾と飛 躍をふくむ衝突、対立による統一、そのための大規模な交流によっての み超えられるであろう。TANIKAWA Gan, 《Sōkan sengen : Sarani fukaku shūdan no imi o » 創刊宣言 さらに深く集団の意味を [Manifeste du premier numéro de la revue : Approfondir le sens d'être un groupe] (S-m, 1-1-3). Les acteurs suivants furent concernés par ces oppositions encouragées : sidérurgistes vs paysans; mineurs vs sidérurgistes; mineurs vs employés de la Caisse d'épargne; mineurs vs habitants de la région d'Aso ; fils de familles paysannes devenus ouvriers vs ceux qui ont repris l'exploitation (les fils aînés) ; contremaîtres vs ouvriers ; titulaires vs sous-traitants ; salariées vs 
noter que les mines de Chikuhō étaient fertiles comme terrain de brassage - et de création -, car à cette époque, un nombre non négligeable de ses mineurs étaient d'anciens soldats, issus de différentes régions du Japon, revenus du continent et dont le parcours fut brisé par la guerre. Ils avaient un niveau d'éducation plus élevé que la moyenne de cette population, d'ordinaire composée de fils cadets d'agriculteurs du sud de Kyūshū et d'enfants de mineurs.

Parmi les participants venant des houillères, on ne connaît aucun travailleur de Mitsui-Miike, le géant industriel implanté dans la région où pourtant des cercles fleurissaient (Cha'en, p. 49). Sākuru-mura recrutait, dans son environnement proche, dans les houillères de taille modeste ou moyenne. Les travailleurs ne bénéficiaient pas des mêmes conditions de travail que les employés des grandes houillères. Ils devaient se résigner à occuper les mines dangereuses ou peu productives qui n'intéressaient pas les grandes entreprises, ou abandonnées par ces dernières. Un ouvrier d'une houillère de taille moyenne de Nakama, au retour de son passage à Miike pour prêter main forte aux grévistes de cette immense structure, écrit : «De toute manière les travailleurs de petites et moyennes mines ne font pas confiance aux travailleurs des grandes mines. "Comme ils sont nombreux, ils ont un important budget de cotisations, alors ils sont arrogants sans jamais participer aux luttes." ». (S-m, 3-5-21) ${ }^{9}$.

À sa sortie, la revue reçoit un accueil enthousiaste. En 1959, elle réussira rapidement à créer un assez vaste réseau et à susciter des débats contradictoires entre différents groupes sociaux, et entre le nord (dit « avancé ») et le sud de Kyūshū (dit « arriéré » mais comportant selon le Village plus de potentiel pour la régénération populaire). En relation avec le secteur culturel du Parti communiste et avec une partie de l'intelligentsia de Tōkyō, elle sera également connue de quelques militants de régions éloignées, et plusieurs tentatives pour créer des mouvements similaires seront annoncées. En même temps, le fossé entre le Village et les grands syndicats des houillères devint définitif dès cette même année 1959.

étudiantes; mines privées vs mines d'État ; éducation nationale vs établissements privés; population autochtone (agriculteurs et pêcheurs) vs nouvelle population (cols blancs) etc. Souvenons-nous qu'en 1956, Mao Zedong lance la « Campagne des Cent Fleurs », encourageant les débats et les critiques du pouvoir.

9. SAKATA Masaru 阪田勝, 《Tōitsu-kyōsen ni igi ari » 統一教宣に意義あり [Je m'oppose à la propagande unique]. 
En 1960 la revue commence à montrer des signes d'essoufflement. À partir du numéro d'avril 1960, elle réduit son nombre de pages. La revue cesse de paraître durant quatre mois après le numéro de mai 1960. Le renouvellement de la convention de sécurité nippo-américaine, mi-janvier, et l'arrêt, début novembre, de la grève qui a duré 282 jours chez Mitsui-Miike frappent de plein fouet le moral des membres. C'est également à cette période que Tanikawa Gan et deux autres membres du Village se voient exclus du Parti communiste, accusés d'être des « ennemis du peuple » (S-m, 3-6-15). Lorsque la revue reparaît en octobre (il s'agit de la seconde série), les caractères d'imprimerie sont abandonnés. Elle est et restera miméographiée. Ni la pagination ni la périodicité ne sont fixes. La revue s'arrêtera soudainement en octobre 1961.

\subsection{Les contributions des fondateurs}

Avant de passer la parole aux mineurs, observons les diverses contributions des trois fondateurs directement liés à la question du charbon. Pour Tanikawa Gan, personnage clef du mouvement, nous ne connaissons pas de texte directement lié à la vie des mineurs, sauf quelques nouvelles non abouties. Il convient plutôt de retenir de lui le fait qu'il a aménagé un espace de langage vivant pour des personnes peu accoutumées à s'exprimer, leur permettant de surcroît d'affirmer leurs différences d'intérêts par rapport à leurs camarades ${ }^{10}$ et de résister à la force centripète de Tōkyō (discours progressistes des intellectuels, du Parti...). Les membres du Village devaient connaître l'appel de ce narodniki japonais, grâce à ses poèmes déjà disponibles à l'époque : «N'allez pas à Tökyō, créez votre pays // dans le salon où la mousse est froide à nos fesses / invitez marins paysans tourneurs mineurs / nos hontes sont innombrables, notre regard unique / c'est ça! la capitale de ce monde cachée sous les fougères $\gg^{11}$.

10. À la marge de Säkuru-mura naîtra même une plaquette féministe, voir à ce sujet la note 14 .

11. 東京へゆくなふるさとを創れ//おれたちの尻を冷やす苔の客間に/船乗り 百姓 旋盤工 坑夫をま放け/かぞえきれぬ䎵辱 ひとつの眼つき/それこそ羊歯 で隠されたこの世の首府. Extrait de 《Tōkyō e yukuna »東京へ行くな [N'allez pas à Tōkyō] (TANikAWA, p. 26). À partir de 1960, Tanikawa déplace son centre d'activité vers Tōkyō où il soutient le mouvement Zengakuren 全学連 (Fédération japonaise des 


\section{CIPANGO}

218 Cahiers d'études japonaises n²4

Tanikawa Gan fut également, à l'arrière-plan, le cerveau dans une lutte à laquelle beaucoup de membres ont participée dans une mine de taille moyenne, après la défaite de Mitsui-Miike. Ce conflit prendra une importance exceptionnelle dans les pages de la revue. Dans le troisième numéro de la seconde série, la rédaction publie son soutien aux travailleurs de la mine Taishō (大正炭鉱, S-m, 3-8-14/15), qui se trouvait à proximité, comme si le Village avait besoin de canaliser là toute son énergie refoulée. Se forment tout d'abord le «Taishō kōdō-tai» (大正行 動隊, groupe d'action pour Taishō), puis le «Kyütan kiki toppa-tai» (九炭 危機突破隊, groupe pour surmonter la crise de la mine Kyūshū) composés de jeunes membres de Säkuru-mura, afin notamment de clamer le refus du rejet des initiatives des minorités. Pour faire entendre des voix dissidentes, ils s'infiltraient dans les rassemblements organisés par les syndicats. Certains d'entre eux ont même réussi à se faire élire à la direction de sections syndicales, mais en même temps une hostilité et des affrontements physiques avec d'autres syndicalistes se multipliaient. De plus en plus de textes prendront la forme de journal d'activiste ; on y retrouve une joie certaine de faire part de leur bravoure. Face à cette escalade, Tanikawa, lui-même victime d'une attaque de jeunes syndicalistes, fut le seul à mener de véritables réflexions. L'une concerne la modalité de prise de décision en démocratie ${ }^{12}$. L'autre réflexion concerne la violence : tout en acceptant d'accompagner les jeunes dans leurs actions directes, Tanikawa a donné deux articles contre la violence (S-m, 3-5-7/9, 4-2-1/5). À cette période déjà, bien avant que l'histoire ne démontre l'impasse à laquelle conduit la spirale des violences intestines, il pose la question de l'engouement pour l'action directe.

Morisaki Kazue est née de parents japonais à Daegu, dans le sud de la Corée sous occupation. Elle bâtira l'ensemble de son écriture sur son trauma d'être venue au monde du côté des oppresseurs. Bien qu'aujourd'hui elle ne reconnaisse plus l'intérêt de Säkuru-mura - sauf pour les écrits de mineurs -, la revue a joué un grand rôle dans la maturation de son écriture. C'est en effet pour cette revue

associations d'autogestion étudiante) avec notamment le critique Yoshimoto Takaaki 吉 本隆明.

12. Le vote à la majorité étant un système qui ne laisse aucune possibilité pour recueillir les voix de la minorité, Tanikawa imagine une démocratie où l'on attribuerait de façon aléatoire un droit de veto à un nombre limité de votants qui ne feraient pas partie de la direction (S-m, 3-8-10/18, 4-2-1/5, 4-5-20). 
qu'elle a composé son premier recueil de témoignages ${ }^{13}$, dans le style appelé kikigaki (聞き書き).

Morisaki choisira d'interviewer des femmes qui ont l'expérience du travail dans les galeries des mines. Il s'agit d'une mémoire sauvée in extremis, puisque le travail des femmes dans les galeries fut interdit en 1947. On y apprend que l'interdiction ne fut pas vécue par les intéressées comme une mesure de protection, mais au contraire, comme un moyen de se débarrasser d'une main d'œuvre peu rentable, devenue excédentaire à cause de la crise. En rassemblant les six articles publiés dans Säkuru-mura, la jeune poétesse publiera en 1961 son premier ouvrage de kikigaki, Makkura : onna kōfu karano kikigaki まっくら 女坑夫からの聞き 書 [Ténèbres: témoignages de fermmes mineuresTénèbres : témoignages de femmes mineures]. Le rôle de la femme dans les galeries était celui d'ato-yama (後山, mont derrière), qui consistait à porter dans un panier les morceaux de charbon extraits par le saki-yama (先山, mont devant) toujours masculin, jusqu'au wagonnet. Il s'agissait d'une forme de travail bien particulière, dans la mesure où le partenariat, formé souvent du père et de sa fille, ou d'un couple marié, pouvait réunir deux personnes totalement étrangères. Quoi qu'il arrive entre les deux, travaillant à demi nus, dans les ténèbres, nul reproche ne leur était fait. Morisaki voyait là une antithèse de la prostituée et de la femme au foyer qui vit sa sexualité confinée dans un rôle d'épouse ${ }^{14}$.

13. Les premiers textes de Morisaki étaient souvent hermétiques, à mi-chemin entre poésie et réflexion. C'est son activité de collecte de témoignages, rédigés dans un langage accessible à tous, qui lui a permis de conquérir un lectorat important. Elle est aujourd'hui connue du grand public comme auteure de reportages, notamment de Karayuki san から ゆきさん [Dames parties en Chine] (1976).

14. Le style kikigaki sera apprécié dans la revue. Faisant suite à cette série, trois kikigaki seront publiés par différents membres, dont « Kibyō : Minamata-wan gyomin no ruporutāju » 奇病 水俣湾漁民のルポルタージュ [Maladie étrange : reportage sur la population de pêcheurs de la baie de Minamata] d'Ishimure Michiko石牟礼道子 (1927-2018, S-m, 3-1-34/48), qui va constituer l'un des épisodes centraux du célèbre Kugai-jôdo 苦海浄土 [La mer de la douleur, le paradis].

Lorsqu'elle décide de s'installer avec Tanikawa Gan, Morisaki Kazue était mariée et avait, comme celui-ci, deux enfants. En août 1959, ayant constaté que les femmes ne peuvent pas s'exprimer, même dans les milieux militants, Morisaki lance depuis sa cuisine un mensuel de fabrication artisanale: Mumei Tsûshin 無名通信[Correspondances anonymes]. Cet 


\section{CIPANGO \\ 220 \\ Cahiers d'études japonaises $\mathrm{n}^{\circ} 24$}

Ueno Eishin a signé plusieurs ouvrages précieux pour ceux qui s'intéressent à la vie des mineurs de cette région. Il était lui-même mineur dans des mines petites et moyennes. Entre 1958 et 1961, période durant laquelle il se charge de Säkurumura, Ueno publie deux ouvrages marquants : Oya to ko no yoru : minna de kaita rakugaki 親と子の夜 : みんなで書いたラク書き [Nuits des parents et enfants : Graffitis écrits tous ensemble] (1959) et Owareyuku kōfu-tachi 追わ れゆく坑夫たち [Les Mineurs mis en fuite] (1960). Le premier regroupe cinq contes illustrés sur la vie des mineurs qu'il avait rédigés. L'auteur y cherche un langage accessible aux premiers intéressés peu habitués à la lecture, et tente de mettre en valeur des moments de félicité dans leur quotidien. Dans « Hitokuwabori »ひとくわぼり [Un seul coup de houe], Ueno met en scène un héros de conte populaire local. En revanche, dans son autre ouvrage Les mineurs mis en fuite, il emploie une écriture de reportage standardisée pour un public habitué à la lecture $^{15}$. Ce livre a contribué à faire connaître le travail dans les mines, au-delà du drame en cours à Chikuhō ${ }^{16}$. Le texte commence par la description de la faillite d'une petite mine. L'auteur y détaille les conditions de travail très éprouvantes,

organe de communication quasi exclusivement féminin marque la gestation de l'une des racines « autochtones » du féminisme japonais.

15. Le livre est du reste sorti dans la collection Iwanami bunko 岩波文庫, la première collection de poche japonaise, créée en 1927 pour permettre à un lectorat de masse l'accès aux ouvrages académiques.

16. «Dans les déserts noirs étalés à perte de vue, avec des lacs sans nombre de toutes tailles formés par des effondrements, dans leurs plis de résidus miniers désagrégés, telles des poux ou des acariens, grouillent, attroupées, des dizaines de milliers de personnes abandonnées. Si vous étiez un voyageur passé pour la première fois, sans aucune connaissance préalable, vous auriez pensé qu'il s'agit d'un cantonnement de tous les sans-abri et clochards du Japon : il n'est pas aisé de reconnaître que cette masse d'innombrables « cadavres vivants » est ce que sont aujourd'hui devenus ces mineurs qui travaillaient jusqu'à hier. 大小無数の陥落湖沼を抱えて果てしもなく広がる黒い砂漠、その風化した ボタ土のひだの間にさながらシラミかダニのごとく蚌いている幾万の棄民 群。もしあなたがそれについての一片の知識も持たないはじめての旅行者 であるならば、まるで日本中のルンペンや乞食を一地域に集結させたかと 思われるほど累々たるこの「生ける屍」の大群が、すべて昨日まで働き続 けてきたあの炭鉱労働者の今日の姿であることを承認するのは、おそら く決して容易なことではありえないであろう。（UENO, 1985, p. 113). Dans le sens où il n'esquisse pas de modèle sociétal à venir dans ses ouvrages documentaires, 
l'errance des travailleurs, l'injustice, l'invalidité, l'indigence, et donne la parole aux anciens mineurs pour leurs récits de vie ${ }^{17}$.

Parmi ses contributions à la revue, il faut citer son reportage publié dans le numéro deux. Intitulé « Retsu » (裂 brisure), ce texte a pour sujet une inondation dans la mine d'Eguchi qui a causéla mort de quatorze travailleurs en 1958. Composé de photos de victimes gisantes, d'écrits de leurs enfants et de « testaments » gravés avec des cailloux sur les casques retrouvés, le récit est particulièrement poignant. Il s'agissait en fait de la republication d'un article rédigé pour la revue Gekkan tanrō 月刊炭労 [Mensuel du Syndicat national des travailleurs des houillères]. Ueno n'y ayant pas caché la tension existant entre les travailleurs de cette petite mine et le Syndicat national, dont les premiers critiquent l'hypocrisie et le bureaucratisme, les passages jugés dommageables au syndicat avaient été censurés. La publication intégrale de l'article par Säkuru-mura signifiait de fait la non soumission de ce dernier au Syndicat national.

\section{Les contributions des mineurs}

Säkuru-mura est riche en textes sur le quotidien des mineurs : travaux, machines, bains, accidents, baraquements au pied de terrils, dettes, 《 désertion 》(ケツ割 り、 ketsuwari), syndicalisme - très nombreuses critiques des cadres -, alcool, familles, enfants... Mais ces images jaillissent le plus souvent de la plume de témoins et rarement des intéressés eux-mêmes, contrairement à ce qu'aurait pu laisser imaginer la contemporanéité de Sākuru-mura avec la vague du « mouvement

contrairement à Tanikawa et Morisaki, la plume militante d'Ueno n'est pas visionnaire. Si l'écriture incarnait une gestation de l'avenir pour eux, celle d'Ueno était un requiem.

17. Matsuura Sōzō et Shibano Tetsuo estiment hautement ce recueil, en le situant dans la lignée du reporter américain John Silas Reed (1887-1920) et de Hosoi Wakizō 細井 和喜蔵 (1897-1925) (MATsuURA \& Shibano, p. 170-171). Ils le comparent même à La Condition ouvrière de Simone Weil (1909-1943) rédigé en 1934 après une expérience de travail dans une usine. Mais les deux écritures apparaissent assez différentes quant au rapport qu'entretient le narrateur avec le terrain de l'observation. La narratrice de Simone Weil préserve une distance avec les personnes à l'entour, tandis que le narrateur d'Ueno se laisse sans cesse pénétrer par le monde qu'il décrit. 
222 Cahiers d'études japonaises nº 24

pour l'écriture au quotidien » (生活綴り方運動 Seikatsu tsuzurikata undō) ${ }^{18}$. Ce genre d'allusions aux difficultés de l'existence nous parvient grâce aux témoignages recueillis par des tiers (comme ceux donnés à Morisaki Kazue par exemple), ou bien encore il s'agissait de fictions. Leurs écrits non fictionnels comprenaient leurs échanges avec les autres groupes sociaux, ou encore, dans une moindre mesure, des réflexions esthétiques. Loin de tomber dans le misérabilisme, les mineurs étaient curieux du monde. Le seul domaine auquel ils n'ont pas pris part est celui de discours abstraits qui proposeraient par exemple un modèle de société vivable.

Par commodité, examinons d'abord les écrits que l'on peut qualifier de non fictionnels au sens large (vie de la revue, débats, récits autobiographiques et reportages), puis les fictions (poésies et nouvelles - toutes sur la vie de mineurs), et enfin une réflexion sur la photographie.

\subsection{Les écrits «non fictionnels 》}

Dans les écrits « non fictionnels », les mineurs ont surtout écrit sur la vie de la revue. En voici un exemple. À peine deux mois après le scandale du reportage d'Ueno, se produisit un autre incident semblable, impliquant cette fois le cercle Littérature Yamada (Yamada bungaku, 山田文学), association littéraire de la mine Mitsubishi de Kamiyamada, dont de nombreux membres étaient des collaborateurs du Village.

Sous la pression du syndicat qui finançait leur publication afin de censurer certains textes, le cercle Yamada bungaku finit par imploser. Village publiera plusieurs textes sur le sujet, dont celui de l'un des acteurs de l'affaire, Kimura Hideo 木村 日出夫, mineur de Mitsubishi-Kamiyamada et membre de Littérature Yamada. Selon son article, le lendemain de la décision de l'arrêt de la publication, alors qu'il descendait dans les galeries, il fut entouré par une quinzaine de mineurs qui n'avaient jamais été intéressés par la littérature auparavant. Ils lui proposèrent de créer ensemble une nouvelle revue littéraire, qu'ils appelleraient Cri de naissance

18. Sauf quelques exceptions dont le poignant «Kōnai dewa shinitakunai ! »坑内では 死にたくない! [Je ne veux pas mourir dans les galeries !] de Matsuoka Yasufumi 松岡 保文 (S-m, 2-4-25/30). 
(Ubugo 産声) ${ }^{19}$. Le Village ne dit pas ce qu'il advint, mais l'anecdote témoigne d'un esprit d'ouverture partagé dans cette mine.

Nous pouvons situer à l'opposé de ces écrits qui collent à l'actualité et sont animés par le militantisme, deux auteurs qui ont publié l'histoire de leur famille. Tomonari Hajime 友成一 n'est pas fils de mineur, et lui-même ne l'est que de façon occasionnelle. Avec la parfaite maîtrise d'un style concis et sobre, il brosse le passé de sa famille autour de la figure du père, souffrant de schizophrénie et séjournant longtemps en prison, accusé à tort d'un viol suivi d'un assassinat. L'histoire est émaillée de maladies, de mésententes et de déménagements ( $\mathrm{S}-\mathrm{m}$ $1-1-14 / 19,1-3-22 / 28)$.

Du reportage aux paroles de chansons, en passant par de nombreux petits encadrés d'actualité, Hanada Katsumi 花田克己a contribué à plusieurs rubriques de la revue. Mais son apport le plus remarquable réside dans ses deux récits de vie. Mineur depuis deux générations, il cherche à comprendre la vie difficile de son père. Plutôt que de ramener le problème au schéma militant contre le pouvoir, il cherche l'origine du mal dans le fait que son père n'était ni le fils ainé, ni le cadet et avait ainsi peu de droit de cité dans ce monde. Le seul patrimoine hérité de ce père, selon lui, était un ressentiment contre la société, mais curieusement cela ne l'a pas empêché d'aimer le métier et de vouloir le transmettre à la génération suivante. Si la vie dans les mines telle que la décrivent les non mineurs échappe rarement au « spectaculaire », leur introspection est discrète et menée avec recul. À travers ces écrits où les scènes se déroulant dans les galeries sont quasiment absentes, on entrevoit le poids du passé invisible dont est chargé celui qui accepte d'y vivre.

Entre les écrits militants et les récits personnels, on peut situer les différents reportages et kikigaki. La curiosité a poussé Hirano Shigeo 平野滋夫 à se promener dans la mine publique Kasuya, gérée par l'État, où il observe des problématiques sociales différentes des siennes (S-m, 2-10-28/32). Kobinata Tetsuya小日向 哲也 ira discuter avec les travailleurs des autres secteurs, écouter la vie d'une vieille dame, bonne (女中, jochū) toute sa vie à Tōkyō (S-m, 1-4-45, 2-11-6/8, 3-2-11/15, 3-8-24/25). Sakata Masaru a même rendu compte d'une visite à un centre de la nouvelle religion Aanai-kyō (ああない教), dans un texte amusé, mais sans jugement (S-m, 3-4-2/5).

19. « Hakkin sono go » 発禁その後 [La suite de l'interdiction], (S-m, 2-2-9/14). 
CIPANGO

224 Cahiers d'études japonaises $n^{\circ} 24$

\subsection{Les fictions}

La plume des mineurs aborde, dans les fictions, exclusivement la vie de la mine. Aucune de ces contributions n'a l'envergure d'un Germinal ou d'un Quai de Wigan $^{20}$, mais certaines révèlent une grande qualité d'écriture. On notera dans l'ensemble des proses de fiction une volonté d'aborder la question du salut dans les mines, en décrivant les tensions créées autour du syndicalisme, en dénonçant les conditions de vie, ou en empruntant le point de vue d'un enfant pour mettre certains moments de l'existence en relief. Dans ce dernier cas, le récit est souvent construit comme un conte pour enfants, invitant à un sommeil rassurant ${ }^{21}$. Il en est ainsi avec la série de contes signé par Kusabue Kensaku 草笛健作 ( $\mathrm{S}$-m, 2-12-24/28, 3-1-23/27, 3-2-29/32).

Dans trois des cinq nouvelles proposées par Yamazaki Kiyoshi 山崎喜与志, les relations avec le syndicalisme offrent le cadre et la trame du récit. L'une des deux autres nouvelles décrit la tension psychologique existant entre un mineur et son jeune fils qui aspire à un autre avenir ; la dernière met en scène une jeune fille de mineur vendue par ses parents pour devenir prostituée. (S-m, 1-3-42/48, 2-6-31/36, 2-10-35/37, 2-11-32/39, 4-5-28/40).

Les mineurs étaient nombreux à proposer des poèmes libres et des tanka ${ }^{22}$. Le genre des vers libre comprenant ses propres difficultés de composition liées justement à l'absence de contraintes formelles, maints textes demeurent dans un état de maturité insuffisant. Néanmoins, on perçoit que ce type de poésie offrait un lieu où prenait forme une pensée. Les motifs de la mort, du sang, du deuil ou de la vermine sont fréquents; le sont moins ceux de la colère et de l'espérance.

Une des rares exceptions est « Taiyō o kajirinagara, Shittai-ninpu wa kataritsuzukeru »太陽を撂りながら 失対人夫は語り続ける [En croquant le soleil - le terrassier bénéficiant de la mesure contre le chômage continue de parler] d'Isayama Masanobu 諌山昌信. Ce long poème fait preuve d'une technicité rhétorique complexe, usant de bribes d'images de la journée d'un ancien mineur militant qui travaille à présent comme terrassier dans le cadre

20. ORWELL Georges, 1937 (Reportage sur le prolétariat des mines).

21. Ce trait délibérément protecteur est partagé par les paroles de chanson composées par les mineurs.

22. Forme poétique ayant comme contrainte la mesure de $5 / 7 / 5 / 7 / 7$ sons. 
d'une mesure d'aide aux chômeurs (失業対策 shitsugyō taisaku). Faute de place pour une analyse approfondie, nous ne pouvons l'aborder en détail. Signalons simplement que dans ce poème l'auteur superpose la narration d'un jeune homme qui cherche à s'installer avec une femme et le moment de négociation passé dans le bureau pour l'emploi. L'image d'un œil invisible surplombe la scène, pleine de désirs et de rage ( $S$-m, 2-6-10/18).

Nous nous contenterons ici de citer un poème d'une écriture plus simple et qui ne nécessite pas de commentaires. «Kyōdai yo nikushimi o »兄弟よ憎し みを [La haine, mes frères !] de Tamaki Makoto 玉木誠, s’apparente à un récit autobiographique ${ }^{23}$.

Jeune,

Père aimait par-dessus tout les geisha et le sake

Il exerçait un pouvoir absolu sur Mère

Sortant des galeries il prenait toujours

Une bouteille de makkari ${ }^{24}$

Tel était mon père.

Mais une seule fois il a bien voulu m'emmener en ville

23. 若い頃/芸者買いと 酒が何よりも好きだった親父/おふくろには絶対的 権力を振るっていた親父/坑内から昇ると必ずマッカリを一升/吞んでいた 親父/そういう親父が/黍を米代わりの粉と換えるため/袋を俺にもたせ/た った一度 町に連れて行ってくれた//その夕方/畳一枚ほどもある硬の下敷 になって/おやじは死んだ/僕は日がたつにつれ/だんだん淋しくなったが/ 葬式の時 あんまり悲しいとは思わなかった/それよりも破れた畳を/大 勢の人から見られるのが厭だった//親父の残してくれたものは何もない/ 坑内で使っていた道具すら返ってこなかった/おふくろは毎日泣いた/もう 一つおふくろが泣いたのは/二番目の兄貴が/アメ公のしょうい弾で殺され た時/引っぱっても引っぱっても 頭を/地面に釷づけにした六角の長い弾 は/抜けなかった/穴のあいた頭に/脱脂綿を三袋も四袋もつめながら/おつ おっ と泣いたのはおふくろ//いままた/三番目の兄貴が落盤で/一生働く ことを取りあげられたが/おふくろに/それら涙を/ただ絶望や悲しみだけで なく/涙の一滴一滴を吞み下し/敵への憎しみに替えることを教えねばなら ない/多くの兄弟に/悲しみを憎しみに替えることを/教えねばならぬ (S-m, 2-6-14/15)

24. Vin coréen. 
Pour échanger notre millet contre la poudre imitant le riz

Me faisant porter un sac.

Ce même soir-là,

Passant sous une dalle de charbon grande comme un tatami

Père a trouvé la mort.

À mesure que les jours passaient

Je sentais qu'il me manquait

Mais aux obsèques pas vraiment la tristesse.

Je détestais plutôt

Que les gens découvrent nos tatami déchirés.

Père ne nous a rien laissé.

Pas même les outils qu'il utilisait dans les galeries.

Mère a pleuré tous les jours.

Une autre fois où elle a pleuré

C'était quand mon deuxième frère aîné

A été tué par une balle incendiaire des yankees.

On avait beau tirer la longue balle hexagonale qui clouait sa tête,

Elle ne s'enlevait pas.

Mère remplissait le trou de la tête

De trois, quatre paquets du coton

En sanglotant.

Et là encore

Par un effondrement mon troisième frère

A été privé à vie de la possibilité de travailler.

Mère

Ses larmes

Je dois lui apprendre à les avaler goutte par goutte

Les transformer

Non seulement en désespoir et en tristesse

Mais en une haine de l'ennemi.

Transformer la tristesse en haine

Je dois l'apprendre à mes nombreux frères. 
La plus importante contribution des mineurs demeure sans conteste leurs tanka, par leur maturité et par leur puissance. La revue a surtout compté trois excellents poètes de tanka travaillant sous terre. Leurs œuvres témoignent du potentiel de cette forme poétique capable de transmettre à la fois une émotion et la situation concrète à son origine.

Un rayon du jour guide

La basse rangée des baraquements

Que traverse l'ombre nette des terrils

OKITA Hiromi 沖田活美 (S-m, 1-1-20) 25

Essuyer le sang sur un pilier de soubassement

Se remplir la bouche d'eau

Pour en vaporiser la blessure

Okita Hiromi (ibid.) $)^{26}$

La foreuse quand on la pousse nos bras mêlés

Haletants

S'amassent nos corps familiers à moitié nus

Okita Hiromi (ibid.) $)^{27}$

Blâmant la lenteur du travail

Le contremaître tout au long de la journée

Reste derrière nous

TAKAMURA Kunio 筀邦雄 $(S-m, 2-1-26)^{28}$

25. あざやかに硬山の投影移しゆく低き屋並みをみちびくひかり Azayakani bota no tōei utsushiyuku hikuki yanami o michibiku hikari

26. 坑木の端にて血糊を拭いとり水をふくみて傷に噴きつけぬ Kōbokuno hashinite chinori o nuguitori mizu o fukumite kizuni fukitsukenu

27. 鈢岩機もろ腕くみて押し合うと息荒げ寄寸親しき半裸 Sakuganki moro ude kumite oshiauto ikiarage yosu shitashiki hanra

28. 捗らぬ作業を罵る職制の一日われらのうしろにつきてHakadoranu sagyōo nonoshiru shokusei no ichinichi warera no ushironi tsukite 
228 Cahiers d'études japonaises n²4

Grève perdue par trahison,

Comme nous sommes pauvres - nous espérons

un parti puissant et organisé

Takamura Kunio (ibid. $)^{29}$

Sale en fin de journée

Un détour manger boire

Tristesse comme une impatience

Takamura Kunio (ibid.) ${ }^{30}$

Réponds sans hésiter

Parle avec fierté

De ton père mort au travail

Takamura Kunio (ibid.) $)^{31}$

Le vent de l'air rejeté emporte

La poussière de la dynamite

À mon souffle mêlée

Yамамото Tsuguru (S-m, 1-1-21) ${ }^{32}$

Le soir j'essuie la poussière de charbon

Déposée le long du jour dans mes yeux

29.うらぎられ敗れしストに貧しければ恃めり組織して強き党 Uragirare yabureshi suto ni mazushikereba tanomeri soshikishite tsuyoki tō

30. 一日を污れて帰れば倚りてゆく飲食は悲し焦燥に似て Ichinichi o yogorete kaereba yorite yuku onjikiwa kanashi shōsōni nite

31. 労働に斃れし父を誇らかに言いて澱みなく人に答えよRōdō ni taoreshi chichi o hokorakani iite yodominaku hitoni kotaeyo

32. 発破煙もわが吐く息も同化されて押し流さる〉排気の風に Happaenmo wagahaku ikimo dōka sarete oshinagasaruru haikino kazeni 
Comme le ferais pour des larmes qui roulent Yамамото Tsuguru (ibid. $)^{33}$

Sous la lumière mange l'air heureux

La petite famille que je soutiens

Moi d'une santé fragile

Yамамото Tsuguru (S-m, 2-1-27) ${ }^{34}$

Dans l'histoire du tanka, la seconde moitié des années 1950 correspond à la période représentée par le Zen.ei tanka (前衛短歌 《tanka d'avant-garde »), expérimental et antiréaliste. Ce mouvement fut précédé par une période où s'écrivaient plutôt des tanka à portée sociale et rigoureusement réalistes, ceux, par exemple, du Shinkajin-shüdan (新歌人集団《collectif de nouveaux poètes »). Les poètes-mineurs ne respectent pas toujours strictement le mètre mais tirent le meilleur parti du réalisme, comme s'ils poursuivaient l'idéal porté dix ans auparavant.

\subsection{Une réflexion critique}

Si aucun mineur n'a été actif dans les débats idéologiques sur la direction du groupe ou les querelles théoriques sur la culture, ils ont été quelques-uns à mener des réflexions concrètes dans les domaines artistiques : la peinture et la littérature.

Ainsi, Ueda Hiroshi 上田博et Shōda Akira 庄田明, deux des artistes (illustrateur / graveur / photographe) qui se chargeaient de la couverture, ont signé ensemble un article, intitulé « Domon Ken no yūki to gosan»土門拳の勇気と 誤算 [Le courage et la méprise de Domon Ken](S-m, 3-4-13/16). Il s'agit d'un dialogue autour du recueil de photos Chikuhō no kodomotachi 筑豊の子どもた $ち$ [Les Enfants de Chikuhō] de Domon Ken 土門拳, édité peu avant. Alerté par

33. 一日溜りし眼の炭塵を取る夕心゙溢れくる涙の如きも拭きて Ichinichi tamarishi meno tanjin o toru yūbe afurekuru namida no gotoki mo fukite

34. 病み易き吾に支へられ灯の下に幸せさうに食ふ小家族 Yamiyasuki wareni sasaerare hino shitani shiawasesōni kuu shō-kazoku 


\section{CIPANGO \\ 230 Cahiers d'études japonaises nº 24}

la situation des mineurs, le célèbre photographe se rend à Chikuhō fin 1959 et réalise cet album en quinze jours. Il sera imprimé sur un papier de mauvaise qualité et vendu à cent yens pour toucher l'opinion publique le plus largement possible. Cent mille exemplaires seront vendus. Avec Hiroshima, celui-ci est aujourd'hui considéré comme un chef d'œuvre du « réalisme social » de l'artiste.

Reconnaissant volontiers la bonne volonté et la technique de Domon, les photographes-mineurs Ueda et Shōda décryptent point par point son approche. Pour montrer la détresse de Chikuhō, son objectif se focalise sur les enfants d'un hameau d'anciens mineurs, notamment sur deux jeunes sœurs dont la mère est partie et le père malade. Comme pour clore cet album désespérant par une touche d'optimise, des photos de la grève de Mitsui-Miike ont été placées à la fin. Les deux populations ayant des intérêts divergents, une victoire de Mitsui-Miike ne serait en aucun cas un salut pour ces enfants. Cette combinaison est donc inacceptable à leurs yeux. Selon Shōda, le rapport entre la réalité de ces enfants et les grandes luttes serait même à l'opposé de ce que Domon avait imaginé. La lutte de MitsuiMiike aurait puisé sa force dans la mentalité communautaire des mineurs précaires (ces derniers ont convaincu une grande partie des travailleurs de Mitsui-Miike de s'émanciper du carcan « politiquement correct » - dirait-on aujourd'hui de Tanrō). Selon eux, le photographe de Tōkyō s'appuie trop sur la compassion envers les faibles car il a été incapable de rendre la complexité et les contradictions du réel, imperceptible en quinze jours, même aux yeux d'un grand photographe. Les mineurs précaires ont une mentalité solidaire et généreuse, et même s'ils ne sont pas organisés, ils continuent à lutter, à s'exprimer, à chercher collectivement des solutions. Leur argument est convaincant et constitue un discours authentique d'opposition, partant de la périphérie.

\section{Conclusion}

Compte tenu des difficultés de la vie quotidienne des auteurs, la richesse de la production des mineurs est tout à fait surprenante. Ces écrits épars témoignent d'une courte période durant laquelle la culture s'offrait aux classes populaires comme un vaste champ du possible, avant de se voir remplacée par une culture de masse et des objets de consommation. 
Toutefois, cela n'explique pas la raison pour laquelle seuls les écrits de Säkurumura ont continué à avoir une influence dans les années 60. Une comparaison avec les cercles littéraires de Mitsui-Miike suggère quelques pistes de réponse. Nous n'avons pu consulter aucune des trente-huit revues littéraires de Mitsui-Miike répertoriées par Cha'en Rika dans « Rōdō-tōsō no naka no bungaku : Miike to bunka-undō » 労働闘争のなかの文学—三池と文学運動 [La littérature au sein des luttes ouvrières : la mine de Miike et le mouvement culturel] ; le seul tanka cité par l'auteur laisse penser que, au moins dans ce domaine, figuraient des auteurs-mineurs de haut niveau ${ }^{35}$. Selon Cha'en, les cercles littéraires de MitsuiMiike avaient comme particularité une recherche de la création collective (offre de paroles de chansons dans les cercles de chorales, séances de lecture mutuelle de textes, etc.), mais ils finirent par se faire absorber progressivement par les revues des syndicats (disparition des revues indépendantes, création de pages littéraires dans les bulletins syndicaux). Le romancier Noma Hiroshi 野間宏 (1915-1991), alors communiste, indique lors d'une de ses visites à Miike que l'occultation des problématiques personnelles pressenties dans les œuvres produites sur place est un danger pour la création collective (Cha'en, p. 56). Mais dans le cas de Säkurumura, où la collectivité était toujours envisagée comme un devenir, ce problème ne se posait pas. Nous avons remarqué dans le présent article la diversité du champ d'intérêts que révèlent les écrits des mineurs, mais la variété de l'ensemble des contributions est encore plus frappante. Le lecteur a souvent le sentiment d'assister au moment où naissent et murissent les idées. C'est sans doute là ce qui différencie les textes du Säkuru-mura des autres publications issues du monde des mines.

Si le Village était relativement libre vis-à-vis du Syndicat et du Parti, il ne l'était pas forcément du regard des quelques intellectuels de Tōkyō qui s’intéressaient à eux. Certains penseurs de la Nouvelle gauche n'ont-ils pas idéalisé Säkuru-mura, du fait que de « vrais travailleurs des mines » leur offraient des textes, tout comme ce même Sākuru-mura a idéalisé le sud de Kyūshū, car il était moins industrialisé ? Le regard de Tanikawa Gan était depuis toujours tourné vers Tōkyō, où il se rendait souvent. Lorsque le poète de «N'allez pas à Tōkyō, créez votre pays »

35. Vingt caisses de charbons chargées au long de la nuit je ne cesse de cracher encore et encore le crachat toujours noir 徹夜して積みたる石炭二十函吐きても吐きても出 づる黒き痰Tetsuyashite tsumitaru sekitan niuppako hakitemo hakitemo izuru kuroki tan. (CHA'EN, 2010, p. 53). 


\section{CIPANGO}

232 Cahiers d'études japonaises nº 24

s'installe à Tōkyō pour s'allier avec Yoshimoto Takaaki et, par la suite, devient cadre d'une entreprise d'édition éducative, de nombreux acteurs du mouvement qu'il avait animé ont dû sentir leur espoir trahi.

Malgré cela, on peut affirmer que Säkuru-mura reste un modèle sans équivalent d'une appropriation populaire d'un vaste champ d'expressions. La lecture des textes écrits par les mineurs brise parfois les idées préconçues sur eux, par leur puissance, leur diversité, leur pudeur et leur profondeur. Il apparaît une part d'opacité dans l'autoportrait du mineur : pourquoi, dans le même sujet, coexistent un sentiment si fort de haine générée par les conditions inacceptables de travail et la fierté et le désir de transmettre son métier, par exemple ? Voilà une opacité, inhérente à toute altérité, qui ne cesse désormais de nous questionner.

\section{Bibliographie}

Amano Masako 天野正子, 2005, Tsukiai no sengo-shi: sākuru, nettowäku no hiraku chiheiつきあいの戦後史一サークルとネットワークが拓く地 平 [L'histoire d'après-guerre des relations humaines, l'horizon ouvert par les cercles et les réseaux], 吉川弘文館 [Yoshikawa-kôbun-kan], 東京 [Tōkyō], 10 p. +286 p. +6 p.

ArAKI Yasutoshi 新木安利, 2010, Säkuru-mura no jiba: Ueno Eishin, Tanikawa Gan, Morisaki Kazue サークル村の磁場 —上野英信、谷川㕍、森崎和 江 [Le champ magnétique du Village des cercles : Ueno Eishin, Tanikawa Gan, Morisaki Kazue], 海鳥社 [Kaichō-sha], 福岡 [Fukuoka], 319 p.

CHA'EN Rika 茶園梨加, 2010, « Rōdō-tōsō no naka no bungaku : Miike to bunka-undō » 労働闘争のなかの文学—三池と文学運動 [La littérature au sein des luttes ouvrières : la mine de Miike et le mouvement culturel], Ritsumeikan gengo bunka kenkyū 立命館言語文化研究 [Etudes de langues et cultures de l'université Ritsumeikan], no 22, 立命館国際言語文化研究 所 Rutsumeikan kokusai gengo bunka kenkyū-jo [Institut international des études de langues et de cultures de l'université Ritsumeikan], 京都 [Kyōto], p. $49-61$. 
KYŪSHŪ SĀKURU KENKYŪ-KAI 九州サークル研究会 [Groupe de recherches sur les cercles de Kyūshū], Sākuru-Mura サークル村 [Le village des cercles], 2006 [1958-1961], 不二出版 [Fuji-shuppan], Tōkyō.

Matsushita Hirofumi 松下博文, 2006, «'Hasamareru' shisō : Sākuru-mura kaisetsu ni kaete »「はさまれる」思想 - 『サークル村』解説にか えて [Une pensée 《 prise entre deux » - en guise de commentaire du Village des cercles], Fuji-shuppan, Tōkyō, p. 5-9.

Matsuura Sōzō 松浦総三, Shibano Tetsuo柴野徹夫, Murayama Kiyohiko 村山淳彦, Uchino Nobuyuki 内野信幸, 1990, Ruporutāju wa sekai o ugokasu : Jon Rido kara gendai e ルポルタージュは世界を動かす： ジョン・リードから現代へ [Les reportages agissent sur le monde : De John Reed à nos jours], 大月書店 [Ōtsuki-shoten], Tōkyō, p. 153-189.

Mizutamari Mayumi 水溜真由美, 2013, Sākuru-mura to Morisaki Kazue： kōryū to rentai no vijon『サークル村』と森崎和江 一 交流と連帯のヴ イジョン [Le Village des cercles et Morisaki Kazue : une vision d'échanges et de solidarité], ナカニシヤ出版 [Nakanishiya-shuppan], Kyōto, 424 p.

SAKAGUCHi Hiroshi 坂口博, 2006, « Sākuru-mura sōkan zenya »サークル 村創刊前夜 [La veille du lancement du Village des cercles], Fuji-shuppan, Tōkyō, p. 16.

TANikawa Gan 谷川雁, 1968, Tanikawa Gan Shishū 谷川雁詩集 [Recueil de poésie de Tanikawa Gan], 思潮社 [Shichô-sha], Tōkyō, 123 p.

Tsurumi Shunsuke 鶴見俊輔, 1976, « Naze sākuru o kenkyū suruka »なぜサ ークルを研究するか [Pourquoi étudier les cercles ?] Kyōdō kenkyū: Shūdan Sākuru no sengo shisō-shi 共同研究 集団サークルの戦後思想史 [Études collectives : Groupes de personnes, L'histoire de l'après-guerre à travers les cercles], 思想の科学研究会 [Shisō no kagaku kenkyū-kai] [Groupe de recherches scientifiques sur la pensée], Tōkyō, p. 5-7. 
CIPANGO

234 Cahiers d'études japonaises nº 24

UENo Eishin 上野英信, 1985, Ueno Eishin-shū 2, Naraku no sei.un 上野英信集 2 , 奈落の星雲 [Recueil d'oeuvres d'Ueno Eishin tome 2, Une nébuleuse dans l'enfer], 径書房 [Komichi-shobō], Tōkyō, 389 p.

YUCHI Asao 湯地朝雄, 1991, Puroretaria bungaku undō : sono risō to genjitsu プロレタリア文学運動 — その理想と現実 [Mouvements littéraires prolétariens : idéaux et réalité], 晚聲社 [Bansei-sha], Tōkyō.

Résumé : Avec la démocratisation de l'après-guerre, le Japon a vu émerger divers mouvements sociaux. C'est le cas des mouvements culturels dits sâkuruundô (mouvements de « cercles »). Pour sa singularité et en raison de son influence chez des intellectuels de gauche des années 1960, certains historiens se sont intéressés à l'un de ces sâkuru, ayant existé entre 1958 et 1961 dans le nord de Kyûshû : le Sâkuru-mura (Village des « cercles »). Son enracinement dans une région houillère s'explique par l'importance de l'industrie minière dans l'histoire des conflits du travail. Des personnes extérieures du monde de la mine s'y sont également investies. Dans ce texte, nous examinerons la réalité des rapports multiples entre les mines et le Sâkuru-mura. Après une brève présentation chronologique, nous aborderons les travaux directement liés à la mine des trois acteurs principaux du mouvement (dont deux non mineurs). Nous analyserons ensuite les textes, nombreux et variés, rédigés par les mineurs afin d'en mesurer l'étendue, et de saisir ce qu'ils donnent à sentir de la vie de la mine.

Mots-clés : années 1960, mouvements sociaux, sâkuru-undô, sâkuru, Sâkurumura, Tanikawa Gan, Ueno Eishin, Morisaki Kazue, Chikuhô, kikigaki, tanka

Abstract: During its democratization process, post-war Japan saw the emergence of a variety of social movements, including the cultural movements called sâkuruundô ("circle" movements). Because of its conceptual novelty, and the influence it had on left-wing intelligentsia in the 1960s, historians are particularly interested in one of them: "Sâkuru-mura (Village of circles)". This movement lasted between 1958 and 1961 in northern Kyûsh $\hat{u}$. Its location in a coal mining area can be explained by the importance of the mining industry in the labor disputes at the time, but it involved 
a number of outsiders also. In this article, we examine the reality of the multiple relationships between the mines and Sâkuru-mura. After a brief chronological introduction, we first focus on how the three leaders of the movement interacted with the mines. Interestingly, two of them were not miners. We then analyze the styles, aesthetics and themes of the numerous and diverse texts written by the miners, in order to understand what they reveal about life in this mining area.

Keywords: 1960s, social movements, sâkuru-undô, sâkuru, Sâkuru-mura, Tanikawa Gan, Ueno Eishin, Morisaki Kazue, Chikuhô, kikigaki, tanka

キーワード：1960年代、社会運動、サークル運動、サークル、サ 一クル村、谷川雁、上野英信、森崎和江、筑豊、聞き書き、短歌 